\title{
Prospective study on the outcome of the sphenoid drill out procedure* $^{*}$
}

\section{Thibaut Van Zele 1 , Bauke Pauwels¹, Frank Dewaele², Philippe Gevaert', Claus Bachert ${ }^{1,3}$}

' Department of Otorhinolaryngology, Ghent University Hospital, Ghent, Belgium

2 Department of Neurosurgery, Ghent University Hospital, Ghent, Belgium

${ }^{3}$ Division of ENT Diseases, CLINTEC, Karolinska Institutet, Stockholm, Sweden
Rhinology 56; 2: 178-182, 2018

https://doi.org/10.4193/Rhin17.078

*Received for publication:

April 20, 2017

Accepted: January 7, 2018

Background: Chronic sphenoid sinusitis refractory to both medical therapy and sphenoidotomy requires a more extended intervention based on the principles of salvage surgery. Our aim is to describe the 'sphenoid drill out'technique as a sphenoid salvage intervention and to outline its implications on clinical outcome and quality of life.

Methodology: 12 patients with chronic sphenoiditis undergoing a sphenoid drill out procedure were examined by nasal endoscopy preoperatively and postoperatively for one year. Preoperative and postoperative quality of life questionnaires (RSOM-31 and SF-36) were obtained.

Results: All but one patient had a completely patent neostium without scar formation. No major complications occurred after this procedure. All patients reported at least an improvement of their symptoms, $50 \%$ of patients were even symptom free at one year after surgery. The median postoperative RSOM-31 score was significantly lower than the preoperative score. Both the physical component summary (PCS) and the mental component summary (MCS) of the SF-36 score improved significantly. None of the patients needed a revision procedure.

Conclusion: Sphenoid drill out is a safe and effective technique with a high success rate. In patients with chronic sphenoid sinusitis refractory to medical therapy and surgery it could be a valid alternative to revision sphenoidotomy.

Key words: chronic sphenoid sinusitis, sphenoid drill out, salvage surgery, sphenoidotomy, endoscopic sinus surgery

\section{Introduction}

Chronic rhinosinusitis is a prevalent disease affecting approximately $5-15 \%$ of the general population ${ }^{(1)}$. Of all sinuses the sphenoid sinus is the least frequent affected. Sphenoid sinus disease usually occurs within the context of pansinusitis ${ }^{(2,3)}$. Isolated sphenoid sinusitis is a rather rare disease entity, affecting approximately 1-3\% of patients with paranasal sinusitis ${ }^{(2,4-7)}$. When medical therapy fails, the next step in the management of chronic rhinosinusitis is usually functional endoscopic sinus surgery (FESS). For the sphenoid sinus this implies an endoscopic sphenoidotomy, which leads to adequate drainage in most patients. Different types of sphenoidotomy have been described. Type 1 which only identifies the sphenoid ostium without further instrumentation, type 2 which implies opening of the sphenoid inferiorly to half its height and upward to the skull base and a type 3 in which the opening is extended to the floor of the sinus and laterally to the vital structures.16

The concept of functional endoscopic surgery is to preserve the pathways of natural mucociliary clearance. This has made an important shift in surgical techniques in the 1980s, with better patient outcomes as a result. However, despite functional surgery and maximal postoperative medical treatment, primary FESS still fails in a relatively large number of patients. It is estimated that approximately $19 \%$ of patients needs revision surgery after primary FESS ${ }^{(1)}$. In these patients it is assumed that the sinuses don't work properly due to loss of cilia function, remodeling changes or predisposing conditions. Loss of cilia function results in accumulation of mucus in the sinus, also called 'the sump effect'. For these patients the concept of salvage sinus surgery has emerged in the last decade. The aim of salvage surgery is 
to reshape the sinus cavity, avoid the sump effect and allow irrigation to replace the mucociliary clearance. This leads to an efficient removal of mucus, debris and crusting and will prevent colonization of the cavity caused by mucostasis. For the maxillary sinus the modified medial maxillectomy has shown to relieve symptoms by avoiding the sump syndrome ${ }^{(8)}$. For the frontal sinus, this implies an endoscopic modified Lothrop procedure or DRAF type III (9). For the sphenoid sinus, in contrast, only few reports have described salvage surgery. Leight et al. suggested a 'sphenoid drill out' procedure as an intermediate step between a sphenoidotomy and a radical sphenoidectomy or marsupialization ${ }^{(10)}$. In this study our aim is to describe the 'sphenoid drill out' technique and its effect on clinical outcome and quality of life for the patients.

\section{Materials and methods}

\section{Patient recruitment}

Twelve patients with unilateral or bilateral chronic sphenoiditis were recruited at the Department of Otorhinolaryngology in the Ghent University Hospital, a tertiary care center. The ethical committee of the Ghent University Hospital, Ghent, Belgium, approved the studies, and written informed consents were obtained from all subjects. Patients' medical history, including symptoms and the number of prior FESS were inquired. All participants were examined by nasal endoscopy preoperatively and postoperatively. Preoperative and postoperative symptom and health questionnaires, including the 31 -item Rhinosinusitis Outcome Measure (RSOM-31) and the 36-item Short Form Health Survey (SF-36), were obtained ${ }^{(11,12)}$. The RSOM-31 disease-specific QOL instrument in which patients are asked to score a list of 31 symptoms and social and emotional consequences, grading them, from 0 to 5 . The product of the severity of the symptom by the importance for each symptom was then calculated and sums were totaled for each patient. The total score range of the RSOM-31 was and we calculated the MCID (Minimum Clinically Important Difference) based on the standard deviation. Basically the RSOM-31 - becomes clinically meaningful when they approximate $1 / 2$ of the standard deviation of the baseline QOL value for the given population.17 For the SF-36, 10 to 12.5 points (100 point scale) represents the minimum change felt to be clinically relevant for diseases such as asthma, chronic obstructive pulmonary disease and coronary artery disease. 17 On the CT scans the width of the sphenoid sinuses was measured and the presence of osteitis was evaluated.

\section{Surgical technique}

The surgical technique of a sphenoid drill out procedure is quite similar to a Draf type III (Modified Lothrop) procedure of the frontal sinuses. The aim is to obtain a common drainage pathway of left and right sphenoid sinuses by enlarging the ostium, removing the intersinus septum and a part of the
Table 1. Descriptives at baseline.

\begin{tabular}{|c|c|c|}
\hline Descriptives & $\begin{array}{c}\text { Mean(SD) } \\
\text { range) }\end{array}$ & $\mathbf{N}$ \\
\hline \multicolumn{3}{|l|}{ Demographics } \\
\hline Age (years) & 54.25 (12.99) & \\
\hline Male & & 4 \\
\hline Female & & 8 \\
\hline \multicolumn{3}{|l|}{ Sphenoid side affected } \\
\hline Bilateral & & 7 \\
\hline Right & & 4 \\
\hline Left & & 1 \\
\hline \multicolumn{3}{|l|}{ Diagnosis } \\
\hline CRSsNP & & 6 \\
\hline CRSwNP & & 3 \\
\hline Fungus & & 2 \\
\hline Pituitary & & 1 \\
\hline \multicolumn{3}{|l|}{ Symptoms } \\
\hline Headache & & 11 \\
\hline Eye symptoms & & 7 \\
\hline Nose symptoms & & 6 \\
\hline Other symptoms & & 3 \\
\hline Fibrosis & & 8 \\
\hline Previous sphenoidotomy & & 8 \\
\hline Number of procedures & 2,12 & \\
\hline \multicolumn{3}{|l|}{ Sphenoid drill-out } \\
\hline with flap & & 3 \\
\hline without flap & & 9 \\
\hline \multicolumn{3}{|l|}{ Adjunctive procedures } \\
\hline Drill-out alone & & 7 \\
\hline Drill-out + Draf III & & 3 \\
\hline Drill-out + other sinuses & & 2 \\
\hline
\end{tabular}

- Eye symptoms: pressure/pain behind the eyes, swelling around the eyes, neuritis optica

- Nose symptoms: rhinorrhea, postnasal drip

- Other symptoms: sensory disturbances N.V, vertigo, ear pressure, dyspnea

posterior nasal septum and rostrum. The procedure is performed under general anesthesia. First, topical vasoconstriction is accomplished by means of a cocaine-adrenaline gauze packing. After identification of the sphenoidal ostium and enlarging the ostia with a straight mushroom punch on both sides, a $U$ shaped incision is made on the posterior part of the septum. A small nasoseptal flap can be raised in the subperiostal plane. Next, the bony posterior 5-10 $\mathrm{mm}$ part of the nasal septum and rostrum is removed which leads to visualization of the intersinus septum. The intersinus septum is then removed as posteriorly as possible with caution, as it can be attached to the internal carotid artery. 

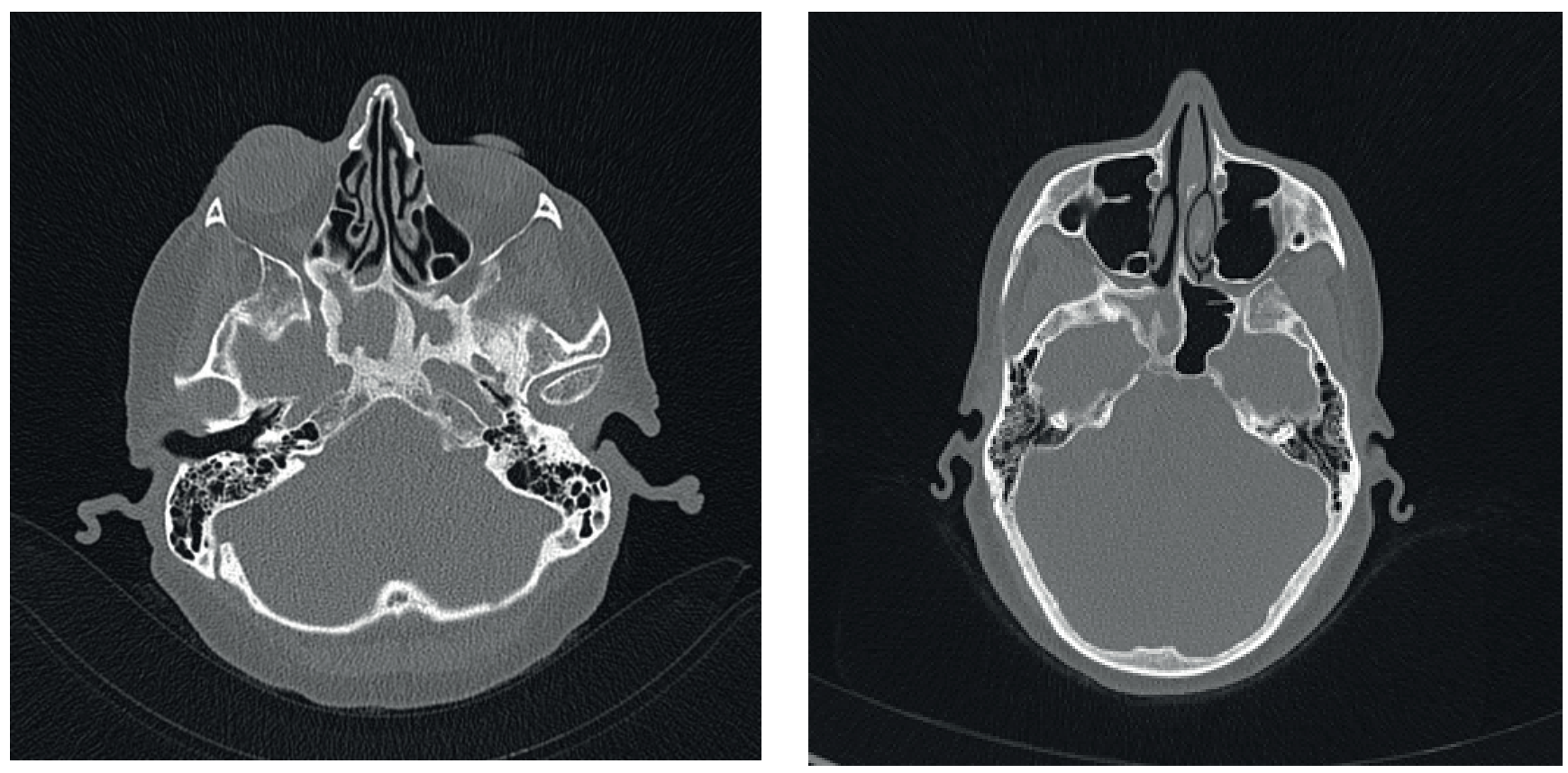

Figure 1. Preoperative CT scan: A: a primary case with bilateral pathology , B: a revision case with unilateral pathology.

The intersinus septum is removed up to the level of the sinus floor. The anterior wall of both sphenoid sinuses are further removed: laterally till the level of the orbital wall, inferiorly up to the level of the sinus floor. If necessary, osteitic bone or diseased mucosa in the sinus is removed. During the procedure different instruments such as the microdebrider, the $4 \mathrm{~mm}$ diamond drill and Kerrison punch are used. This technique can be extended with the creation of a small nasoseptal flap which can be positioned on the floor of both sphenoid sinuses. Postoperatively during the first for weeks all patients used routinely saline douchings and ointment 4 times daily, a nasal corticosteroid spray twice daily and if indicated culture directed antibiotics for 10 days. Patients were seen every week during the first months for endoscopic suction cleaning and debridement.

\section{Statistical analysis}

The Statistical Package for the Social Sciences (SPSS statistics version 22) was used for the statistical analysis. The preoperative and postoperative RSOM-31 and SF-36 scores were analyzed using the Wilcoxon signed rank test. Data are expressed as median and standard error of the mean (SEM).

\section{Results}

A total of twelve patients underwent a sphenoid drill out procedure, of which 8 men and 4 women (Table 1). The mean age was 54,25 years. $6 / 12$ patients were diagnosed with chronic rhinosinusitis without nasal polyposis, 3 had chronic rhinosinusitis with nasal polyposis, 2 patients had fungal disease and one patient needed sphenoid surgery because of scar formation after a former intervention for a pituitary macroadenoma. In 7 patients sphenoid disease was bilateral, in 4 patients the right sphenoid sinus was affected and in 1 patient the left sphenoid sinus (Figure 1). The most reported symptom was headache (11/12). Eye symptoms, including pressure or pain behind the eyes and swelling around the eyes, were reported in 7 of the cases. In 6 patients nasal symptoms such as rhinorrhea and postnasal drip were also present. In 8 patients there was associated fibrosis and in 6 there was presence of osteitis, with a mean global osteitis score of $4.0(\mathrm{SEM}=0.50)$ and a mean osteitis score according to Lee et al. of $2.0(\mathrm{SEM}=0.17)^{(13,14)}$. Patients had a mean of 2,12 prior functional endoscopic sinus surgery, none of them had a prior sphenoid drill-out procedure. In 3 of the drill-out procedures a flap was used. 7 patients had a drill-out alone, 3 also had an associated Draf type III procedure and 2 were also operated on other sinuses. No complications including epistaxis or major injuries to the optic nerve, vidian nerve or internal carotid artery occurred during this procedure.

The mean length of follow-up was 14,25 months (Table 2). All but one patient had a completely patent neostium without scar formation on their last control visit (Figure 2). Only one patient had a partially obstructed neostium with moderate inflammation of the mucosa.

None of the patients needed a revision drill-out procedure so far. All patients reported at least an improvement of their symptoms, half of the patients were even symptom free (Table 2). The mean preoperative RSOM-31 score was 10.55 ( $\mathrm{SD}=3,4)$; the mean postoperative RSOM-31 score was 5,90 (SD 3,67)), which is significantly lower $(p=0.002)$. The MCID was calculated as half of the SD of the baseline value, which was 1,72 . The difference between preoperative and postoperative RSOM-31 values 
Table 2. Outcome measures and data.

\begin{tabular}{|lll|}
\hline Outcome & \multicolumn{1}{c}{ Mean (SD) } \\
\hline Follow-up (months) & $14.25(9.8)$ & \\
\hline Revision drill-out & & 0 \\
\hline Nasal endoscopy & & 11 \\
\hline Neostium completely patent & & 1 \\
\hline Neostium partially patent & & 1 \\
\hline Mucosal inflammation & & \\
\hline Symptoms & & 6 \\
\hline No improvement of symptoms & & 6 \\
\hline Improvement of symptoms & & \\
\hline Symptom free & & \\
\hline Quality of life questionnaires & & \\
\hline Preoperative RSOM-31 & $10.55(3.45)$ & \\
\hline Postoperative RSOM-31 & $(5.9(3,67)$ & \\
\hline RSOM-31 difference & $-4.64(2.89)$ & \\
\hline Preoperative SF 36 total & $76.23(26.88)$ & \\
\hline Postoperative SF36 total & $111.92(37.52)$ & \\
\hline SF36 total difference & $35.69(32.92)$ & \\
\hline Pre SF-36 physical & $37.41(13.53)$ & \\
\hline Post SF-36 physical & $55.29(21.82)$ & \\
\hline SF-36 physical difference & $17.88(19.12)$ & \\
\hline Pre SF-36 mental & & \\
\hline Post SF-36 mental & & \\
\hline SF-36 mental difference & & \\
\hline & & \\
\hline
\end{tabular}

was $-4,64$ and exceeds the calculated MCID.

The SF-36 score is divided in a physical component summary (PCS) and a mental component summary (MCS). The mean total preoperative SF36 score was 76,23, the The mean preoperative $\mathrm{PCS}$ was 37,41 , the mean preoperative MCS was 38,82 . Postoperative total, PCS and MCS were 111,92, 55,29 and 56,63 respectively. Both these scores were significantly higher than the preoperative scores ( $p=0.004$ and $p=0.010$, respectively) (Graph 1). Also for the SF36 the difference between pre-and postsurgery ( 35,69 points) exceeds the MCID ( 12,5 points) and is considered as clinically relevant.

\section{Discussion}

Chronic sphenoid sinusitis refractory to both medical therapy and sphenoidotomy is not common. In these patients a more extended intervention, based on the principles of salvage surgery, is needed. Until recently, literature on salvage surgery was scarce ${ }^{(15)}$. It is only in the last two decades, with the evolution in functional sinus surgery and instrumentation for skull base surgery, that literature on the subject is growing. At present salvage surgery for maxillary and frontal sinuses, such as a mo-
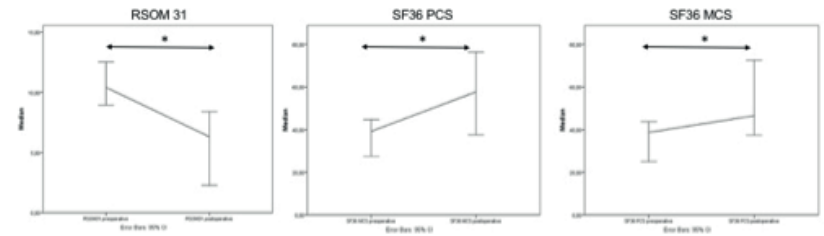

Graph 1. Pre- and postoperative RSOM-31 and SF-36 scores.

dified medial maxillectomy and a Draf III procedure, are already well known and comprehensively described in literature ${ }^{(8,9)}$. For the sphenoid sinus, however, only few reports have described salvage surgery. In this study we present the sphenoid drill out technique as an intermediate procedure between sphenoidotomy and radical sphenoidectomy or sphenoid marsupialization ${ }^{(3)}$. A sphenoid drill out differs from a radical sphenoidectomy in that the latter also includes total removal of the sphenoid floor, creating a continuum with the nasopharynx. This, however, requires extensive drilling which makes a radical sphenoidectomy an aggressive and time consuming procedure.

This study demonstrates that the sphenoid drill out is a safe and effective technique. There were no complications and the clinical symptoms improved significantly in all patients. The procedure has a high success rate; in none of the patients there was a need for revision surgery within the first year. Objective quality-of-life measures were obtained by means of the validated RSOM-31 and SF-36 questionnaires to better understand the implication for the patients. With significant changes of both scores postoperatively we could demonstrate that the sphenoid drill out intervention has a positive effect on the patients' quality of life.

The results of this study demonstrate that, being a less time consuming intervention, the sphenoid drill out might be a better alternative to a sphenoid marsupialization. In that regard, the latter procedure can be preserved only for the most refractory cases. It is our opinion that this technique is still a salvage procedures which should be reserved for revision cases and should not, or rarely be performed as a primary technique. The indications for which this procedure was offered to patients were multiple previous revisions of sphenoidotomies or cases with a very narrow osteitic sphenoid sinus. Although no complications occurred in our series, surgeons should keep in mind the potential serious complications when performing surgery in and around the sphenoid sinus as bleeding of the sphenopalatine and internal carotid artery, damage to the optic nerve, sella or skull base.

A shortcoming in this work is the small subset of patients. Considering the low prevalence of refractory chronic sphenoiditis, recruitment of larger study populations might be the challenge for future research. 

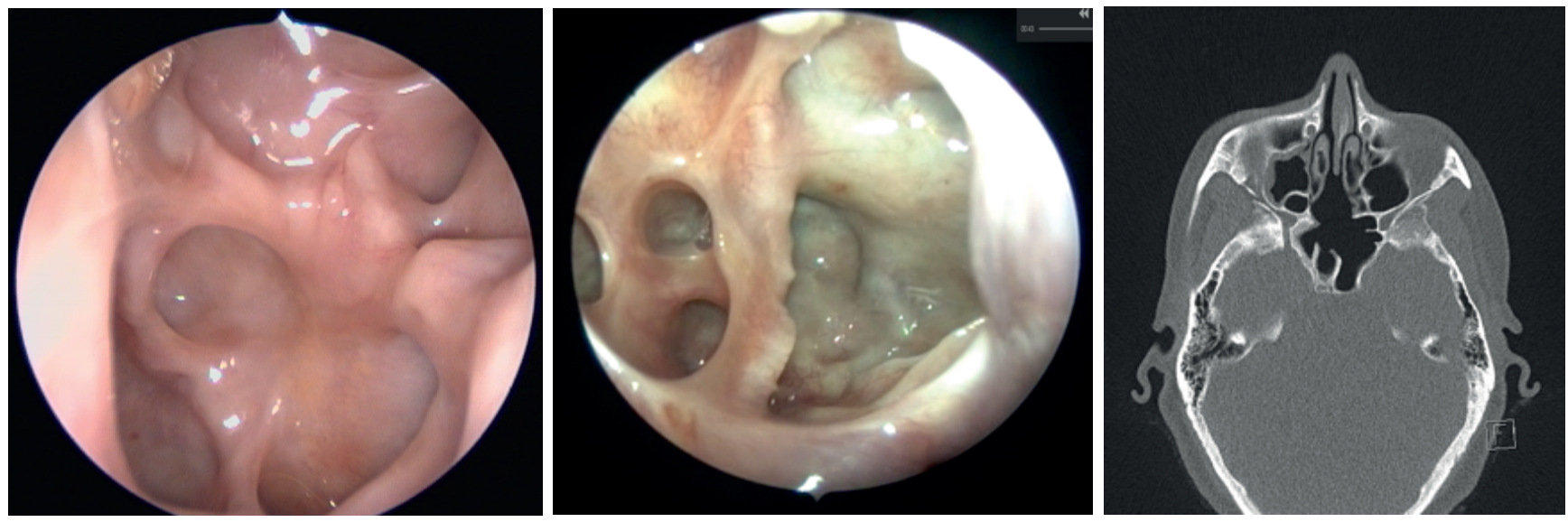

Figure 2. Postoperative endoscopic image, A) a primary case with bilateral pathology, B) revision case with unilateral pathology, C) postoperative CT scan case with unilateral pathology (4 months).

\section{Acknowledgements}

This research did not receive any specific grant from funding agencies in the public, commercial, or not-for-profit sectors.

\section{Authorship contribution}

TVZ designed the study, selected the patients and performed all surgeries. Postoperative follow-up was conducted by TVZ,
CB and PG. TVZ and BP contributed to the acquisition, and CB additionally to the analysis and interpretation of data. TVZ and $\mathrm{BP}$ wrote the manuscript. All authors have critically revised the manuscript and approved the final version of the manuscript.

\section{Conflict of interest}

The authors declare that there is no conflict of interest.

\section{References}

1. Bachert C, Pawankar R, Zhang L, et al. ICON: chronic rhinosinusitis. World Allergy Org. J. 2014; 7(1): 25

2. Pearlman SJ, Lawson W, Biller HF, Friedman WH, Potter GD. Isolated sphenoid sinus disease. Laryngoscope. 1989; 99(7 Pt 1): 716 20

3. Donald PJ. Sphenoid marsupialization for chronic sphenoidal sinusitis. Laryngoscope. 2000; 110(8): 1349-52.

4. Wang ZM, Kanoh N, Dai CF, et al. Isolated sphenoid sinus disease: an analysis of 122 cases. Ann Otol Rhinol Laryngol. 2002; 111(4): 323-7.

5. Castelnuovo P, Pagella F, Semino L, De Bernardi F, Delu G. Endoscopic treatment of the isolated sphenoid sinus lesions. Eur Arch Otorhinolaryngol. 2005 Feb;262(2):142-7.

6. Ruoppi P, Seppa J, Pukkila M, Nuutinen J Isolated sphenoid sinus diseases: report of 39 cases. Arch Otolaryngol Head Neck Surg. 2000 Jun;126(6):777-81.

7. Lawson W, Reino AJ. Isolated sphenoid sinus disease: an analysis of 132 cases. Laryngoscope. 1997; 107(12 Pt 1): 1590-5.

8. Thulasidas P, Vaidyanathan V. Role of modified endoscopic medial maxillectomy in persistent chronic maxillary sinusitis. Int
Arch Otorhinolaryngol. 2014 Apr;18(2):15964.

9. Naidoo $Y$, Bassiouni A, Keen M, Wormald PJ. Long-term outcomes for the endoscopic modified Lothrop/Draf III procedure: a 10-year review. Laryngoscope. 2014; 124(1): 43-9.

10. Leight WD, Leopold DA. Sphenoid "drillout" for chronic sphenoid rhinosinusitis. Int Forum Allergy Rhinol. 2011 JanFeb;1(1):64-9.

11. Ware JE, Jr., Sherbourne CD. The MOS 36-item short-form health survey (SF-36). I. Conceptual framework and item selection. Med Care. 1992; 30(6): 473-83.

12. Piccirillo JF, Edwards D, Haiduk A, Yonan C, Thawley SE. Psychometric and Clinimetric Validity of the 31-Item Rhinosinusitis Outcome Measure (RSOM-31). Am J Rhinol. 1995; 9(6): 297-306.

13. Georgalas C, Videler W, Freling N, Fokkens W. Global Osteitis Scoring Scale and chronic rhinosinusitis: a marker of revision surgery. Clin Otolaryngol. 2010 Dec;35(6):455-61.

14. Lee JT, Kennedy DW, Palmer JN, Feldman M, Chiu AG. The incidence of concurrent osteitis in patients with chronic rhinosinusitis: a clinicopathological study. Am J Rhinol. 2006; 20(3): 278-82

15. Schlosser RJ. Surgical salvage for the non- functioning sinus. Otolaryngol Clin North Am. 2010; 43(3): 591-604, ix-x.

16. Simmen D, Jones NS. Manual of Endoscopic Sinus Surgery. Thieme; 2011

17. Wyrwich KW, Tierney WM, Wolinsky FD. Further evidence supporting an SEM-based criterion for identifying meaningful intraindividual changes in health-related quality of life. J Clin Epidemiol. 1999:52(9):861-873.

Thibaut Van Zele, MD, PhD

Department of Otorhinolaryngology Ghent University Hospital B-9000 Ghent

Belgium

Tel: 003293322332

Fax: 003293324993

E-mail: thibaut.vanzele@uzgent.be 